\title{
Trends and Gaps
}

\author{
Alan Dix, Benjamin Weyers, Judy Bowen, Philippe Palanque
}

\begin{abstract}
This chapter attempts to identify future research directions for formal methods in HCI. It does this using two main approaches. First we will look at trends within HCI more broadly and the challenges these pose for formal methods. These trends in HCI are often themselves driven by external technical and societal change, for example the growth of maker/hacker culture and the increasing dependence of digital technology for basic citizenship, effectively establishing external requirements for the field. Second we will look inwards at the FoMHCI literature, the user interaction phenomena it is trying to address and the processes of interaction design it is intended to support. Through this second analysis we will identify internally generated trends. This does not lead to a single overarching research agenda, but does identify a number of critical areas and issues, and hence establishing opportunities for further research to expand the state of the art.
\end{abstract}

\section{Introduction}

This book attempts to capture a snapshot of the state of the art of research in formal methods in human computer interaction. In this chapter, we ask where it is going and where it could go. Where are the gaps, the opportunities and the challenges for the next decade?

The first part of the chapter looks outward, at broader changes in HCI, how technology and role of technology is changing, from big data and the internet of things to the pivotal role of information technology in modern society.

The second looks more inward at the role of formalism, which aspects of user interaction are being studied and modelled, how formal methods fits into and is addressing different parts of the design and deployment process, and how the techniques and methods within formal methods in HCI are changing.

For each issue we attempt to identify the challenges this poses for formal methods research in HCI and so create a road map, especially for those starting in the field looking for open questions and avenues for theoretical and practical research.

Alan Dix

Talis, UK; University of Birmingham, UK. email: alan@hcibook.com 


\section{HCI trends}

The roots of HCI can be traced back many years, indeed the first true HCI paper was Brain Shackel's 'Ergonomics for a Computer' in 1959; however, the discipline formed properly in the 1980s including the foundation of many of the major international conferences. The study of formal methods in HCI can be traced back to this same period including Reisner's (1981) use of BNF, Sufrin's (1982) Z specification of a display editor and the first PIE paper (Dix and Runciman, 1985).

This flowering of HCI research of all kinds was closely aligned to the growth of the personal computer, which moved computing from the domain of a few technical specialists behind sealed doors, to ordinary professionals on their desktops. In the intervening thirty-five years both technology and the use of technology have changed dramatically. Many of the old concerns are still important today, but there are also new directions in HCI and these create new challenges for the use of formal methods.

In this section, we'll trace some of those trends based largely on a recent JVLC article that examined future trends of HCI (Dix, 2016). We will then use these general HCI trends to highlight some of the formalisation challenges they present. The trends are divided into three kinds: changing user interaction, changing technology, and changing design and development. Of course, these are not independent; indeed, as noted, the whole discipline of HCI effectively grew out of a particular technological change, the introduction of the desktop computer, and this close interplay between technology and use has continued. While available technology does not necessarily determine the use of that technology, it certainly makes new things possible.

\subsection{Changing user interaction}

The use of computation has evolved from the desktop to virtually every aspect of our day-to-day lives.

Choice and ubiquity - In the early days of HCI, computers were largely used as a part of work, your job and employer largely dictated whether you used a computer and, if so, the hardware or software you used. The justifications for usability were therefore essentially about the efficiency of the workforce. The rise of home computing in the 1990s, and particularly the growth of the Internet in the early 2000s, meant that the range of users was far wider and furthermore the users were customers and had choice - if applications were not usable and enjoyable they would be rapidly dumped! This process has continued as process and form factors have made computing (in some form) available to ever-wider groups worldwide. However, in recent years, this ubiquity has meant that computer access 
is assumed. In commerce, internet shopping is not only commonplace, but it is usually the cheapest way to obtain many items; for example, airport checking is often very expensive. In civic society, eGovernment services are not only common, but some countries are seeking to make them the only way to access certain services; for example in the UK so called 'universal benefits', which integrate many kinds of different welfare payments, can only be accessed via an internet portal, despite low levels of digital literacy among precisely the social groups who are likely to be claimants (Citizens Advice Bureau, 2013; Sherman, 2013). That is computer, and in particular internet, services are beginning to underpin society, so that digital exclusion becomes social exclusion; use is no longer a choice but a necessity for participation in civic society.

Formal challenges: The change from optional to necessary use of digital services makes it more important to be able to deal with all kinds of people and also limited digital access. One of the strengths of formal methods is that it can help us analyse and design for situations and people we do not naturally experience or understand. The way this works out people and for devices and contexts are explored in the next two trends.

Diverse people - HCI has always had strands that focuses on those who are different from the 'norm' those with varying abilities and disabilities, different cultures or different ages. Of course, this 'norm' has been contested; for example, the tendency to use undergraduate students as the principle subjects in experiments has meant that what is assumed to be universal human behaviour turns out to be very biased towards western culture (Henrich et al, 2010). Proponents of universal design have long argued that we are all 'disabled' under certain circumstances, for example, effectively blind to dials and controls while our visual attention is on driving, and therefore that if design that is good for those with some form of perceptual, motor or cognitive disability is in fact design that is good for all. Although, for many years forms of anti-discrimination legislation have made 'design for all' mandatory, it has still remained a marginal area.

This has long been problematic, but now, because computation is becoming essential for day-to-day life, it is impossible to ignore. The necessity of access combined with aging populations in many countries means that universal access is now essential. This is exacerbated in many countries where aging populations mean that some levels of perceptual, motor or cognitive impairment is now 'normal' and universally where broadening societal use includes those with low digital literacy and indeed low literacy including the so called 'next billion' users in the developing world. Increasingly, we need to consider those at the social, geographic and economic margins, not just the professional 'class A/B' users of the 1980s.

Formal challenges: Within the formal methods community, work on multimodal systems and various forms of model-based interfaces (e.g. Chap. 18; Coutaz, 2010; Meixner et al, 2011), go some way to addressing these issues. In professional development internationalisation is normal practice for product delivery; and ability checklists are used to tune technology to individual abilities 
(Dewsbury and Ballard, 2014; Whittington and Dogan, 2016). The latter are often 'formal' in the sense that they have codified knowledge, but whereas most work on formal methods is based around relatively complex analysis of relatively simple specifications; practical development has relatively large corpora of codified knowledge, but with very simple, tick-box-style reasoning.

There are clear opportunities to create user models that encompass the wide variations in human abilities, and formal technical challenges to combine the kinds of codified knowledge already available with other forms of formal reasoning.

Diverse devices and contexts - The mobile-first design philosophy has for some time emphasised that for majority users mobile devices may be their principle, or in the case of the 'next billion' possibly first and only, access to computation. Commercially the growing range of devices commonly used now includes smartphones, tablets, smart TV, game consoles, and public displays as well as various forms of laptop or desktop computers. Perhaps as important, when we look at need for universal access 'at the margins', we have to consider poor network connectivity, 'last generation' technology, and in many areas intermittent or total lack of power.

Formal challenges: The challenges of designing for multiple devices are being dealt with fairly well, both in the formal community with work on plasticity and professional practice, notably responsive design. More generally, this suggests we need methods that model both device characteristics, and their environment. Chapter 12 is a good example of the later capturing interactions between environmental aspects such as noise, with device modalities, such as audible output. It would be good to see these areas of research expand, in particular to include infrastructure context such as limited networks or power, not just screen size. We often do not even have adequate vocabulary for these, for example, rural networks often experience frequent short glitches, complete drops in connectivity for a few seconds or minutes; with no formal metric for these drops adequate service cannot be specified in the way that bandwidth or latency can. Theoretical work in these areas may have very immediate practical benefits; despite the widespread focus on responsive design, it is common to have failings such as drop-down menus that do not fit on small-screen devices, and even when executed well, responsive design rarely breaks the mould of simple screen size, rather than more radical modification of the interaction style to fit the device and infrastructure context.

Physicality and embodiment - One of the defining features of early user interface design was the identification of key abstract interaction primitives, not least the windows, icons, menus and pointers of WIMP. Having these abstractions made it possible to easily design applications in the knowledge that while the details of how these primitives appear and behave may vary between specific devices, they can be assumed to exist and in some ways isolate the application from the vagaries of specific devices and even operating systems. On the other hand, as 
Apple have exploited particularly well, there has always been a close relationship between physical design and software design.

In more recent years various factors have made the physical, embodied and situated nature of digital technology more significant. Some of this is connected with new interaction modalities such as bodily interaction with Kinect or geo-spatial interaction such as Pokemon Go. Ultra-haptics now means it is even possible to give holodeck-like mid-air haptic feedback (Carter et al, 2013). In addition, as computation has become embedded in everyday objects and the environment, it becomes hard to separate the digital and physical design: this is evident both in research fields such as tangible user interfaces (Ishii, 2003) and ubiquitous computing (Weiser, 1991), as well as practical design such as screen-based washing machines, or public displays.

Formal challenges: The abstraction offered by WIMP has been helpful in formal specification, which has typically been able to operate well above the physical interaction layer of ARCH/Slinky (UIMS, 1992; Gram and Cockton, 1996). There is some work that deal with the more physical nature of devices including Eslambolchilar's (2006) work on cybernetic modelling of human and device interactions, Thimbleby's (2007) work on physical control layout, physigrams as described in Chapter 9 in this volume, and the use of space syntax and other formalisms for movement in the environment (Fatah gen Schieck et al., 2006; Pallotta et al., 2008). However, compared with more abstracted user interface specification, this work is still nascent.

Really invisible - Weiser's (1991) vision of ubiquitous computing has computers becoming 'invisible'; however, this was in the sense that there are displays everywhere at various scales, but we are so used to them, they fade into the background. This is clearly happening, indeed it is an interesting exercise to walk around your house and count the displays. However, not all computers have obvious displays, and yet this computation embedded into the environment is becoming ever more common (e.g. a modern train has many hundreds of computers in each carriage controlling everything from lighting to toilet doors). Sometimes there is an explicit non-visual user interface, such as body interaction to control a public display or Star Trek style voice commands. Sometimes there may be more implicit sensing leading to apparent effects, such as a door opening or light coming on. Some sensing may operate to facilitate user interactions in ways that are far less apparent, including the low-attention and incidental interactions described in Chapter 7.

Formal challenges: There has been some formal methods work dealing with the architectural design of this form of environmentally embedded system (e.g. Bruegger, 2011; Wurdel, 2011), some (e.g. Chap 7) dealing with non-UI interactions, and some including models of the physical environment (e.g. Chaps $7,8,9,12,15)$. However, like the related area of physical interactions, this work has nothing like the maturity of more abstracted direct interactions. 
Experience and values - The shift in the 2000s from the professional to the domestic domain and the associated shift from employer decision to consumer choice meant that 'satisfaction' the oft-ignored lesser sibling of 'effectiveness, efficiency and satisfaction' began to take centre place. The most obvious sign of this was the job title changes from 'usability' and 'interaction design' to 'user experience'. However, this change in job title represented a more fundamental shift in focus towards the aesthetic and emotional aspects of design (Norman, 2005). Furthermore, increasing scrutiny of the ways in which user interfaces permeate commercial and societal life, has led to an examination of the ways in which values are purposefully or accidentally embodied in designs (Cockton, 2004; Harper et al, 2008).

Formal challenges: While important trends, it is less clear, given the current state of knowledge, how these issues can be dealt with in a more formal way. One potential path might be to enable forms of annotation and argumentation around designs. Notations such as QOC or gIBIS allow the formalisation of argumentation structures, even though the semantic content of the arguments is entirely captured in textual labels and descriptions. As well as offering potential ways to document and track emotional and value aspects of a design, encouraging the user experience designer to create more formal descriptions, could allow automated analysis of more workaday aspects of usability.

Social and personal use - Communication has always been a core part of computer use, from simple email to rich collaborative work; however, the growth of social networking has changed the dominant kinds of communication from functional to phatic. Furthermore, individual use of computers is often very personal, not least the collection of data related to heath and well-being. From an interaction point of view, the focus is, as in the last issue, more about communicating feelings then information. From a governance point of view, there are increasing worries abut the way information and images once shared cannot easily be recalled, the potential for abusive interactions, and the ways in which data analysis can be used by commercial and government bodies in ways which we had never imagined when simply posting a Tweet.

Formal challenges: Several of the chapters in this book include multiple actors (e.g. Chaps 13,15), but dealt with largely in terms of the functional effects of their interactions. There has also been work formalising collaborations in terms of beliefs (e.g. Ellis, 1994) including the way this could be used to make sense of certain artistic installations (Dix et al, 2005). The most extensive formal work on the actual social aspects of interaction are in social network analysis, but to date this is entirely separate from interface-level analysis.

In the area of personal data, the earliest paper on privacy in HCI included simple formalism (Dix. 1990), and there have been multiple works on privacy preserving frameworks and, perhaps most relevant, ways of exposing the implications of data sharing (Langheinrich, 2002; Hong and Landay, 2004). Issues of authentication, security and provenance are heavily formalised, however, as with the more 
social aspects, to date in ways which are largely disjoint from user interface specification and analysis.

Notification-based interaction - Most social network applications are strongly oriented around streams and notifications. These shift the locus of control away form the user and to the choices that the system makes of what to show and when to make it known. There is concern that this can lead to loss of focus and efficiency, indeed a survey of American college students found that more than $90 \%$ reported that digital technologies cause some distraction from their studies, and $34 \%$ more serious distraction (McCoy, 2016), and another study found that in-class cell phone use (presumably for texting) led to a drip of a 1/3 of a grade point (Duncan et al, 2012).

Formal challenges: The majority of formal user interface specification techniques are oriented around explicit user controlled interaction with only a small amount of work in the formal domain on interruptions (Dix, Ramduny-Ellis and Wilkinson, 2004) and dealing with interactions at difference paces (Dix, 1992b). However, there is extensive non-formal literature on the impacts of interruptions and multi-tasking (Adamczyk and Bailey, 2004; Czerwinski et al, 2004; Bailey and Konstan, 2006) and the opportunity to find ways to match the pace and timing of delivery of notifications to the user's tasks (Dix and Leavesley, 2015).

Basic HCI - Although we have had over thirty years of 'standard' usability, still we do not get it right! For example, Apple is often seen as the pinnacle of design, yet, when you turn on a MacOS or iOS device, the splash screen invites interaction (password for MacOS, slide to unlock for iOS) well before the system is ready to interpret your actions. Some of this is probably due to the shift of foci towards aesthetic and emotive design; some to do with the very success of user interfaces meaning more and more people are creating web interfaces in particular, but with less intense training and background than was once the case.

Formal challenges: Tool support could help this and indeed several of the chapters $(17,18)$ describe tool suites that aid designers (although in some cases quite engineering-savvy ones) to develop and analyse user interfaces. Work clearly needs to be done still to improve both (i) the level and kinds of analysis so that they can truly be used as expert guidance for the novice; and (ii) be targeted so that a designer without a formal/mathematical background can use them. The domain specific modelling notations described in Chapters 5 and 16 are one approach to achieving this. 


\subsection{Changing technology}

New and emerging technologies pose fundamental challenges for people and society, with corresponding challenges for formalisation.

Vast assemblies - Smartphones users may have many dozens, even hundreds of apps, albeit the majority of interaction is with only with a few. In the physical world, the Internet of Things (IoT) promises to fill homes and workplaces with large numbers of potentially interacting small devices. Users will need means to manage and configure these devices, understanding how they can work together to solve specific problems. The larger the collection of devices or apps, the harder this will become. Furthermore, these vast assemblies of small items are likely to suffer from feature interactions, that is where two features, each potentially valuable in their own right, interact badly together. For example, imagine that your kitchen smoke detectors are programmed to increase their sensitivity when they detect the house is empty as no cooking is expected; however, if the internet enabled kettle turns itself on a few minutes before you arrive back from work, the steam may well set off the fire alarm.

Formal challenges: Dealing with large numbers of simple objects seems like ideal territory for formal methods. On the configuration side, Chapter 16 shows how workflow notations can be used to connect together apps to make larger functionality; this could be combined with techniques to infer or tangibly programme connections (Turchi and Malizia, 2016). Feature interactions have been studied for many years in telecoms, so there should be knowledge that could be borrowed and modified to deal with other kinds of complex assemblies.

Big Data - Various forms of big data have been the subject of government funding, popular press and of course extensive commercial interest; this ranges from from social networks, as discussed above, to large-scale science, such as at CERN. User interfaces to analysis tools and visualisation have some novel features, but in some ways not so dissimilar to relatively long-standing work in visualisation, data analysis and visual analytics (Thomas and Cook, 2005; Keim et al, 2010). However, the vast scale does introduce new issues: how to get an overview of data that is too big to scan, how to track ownership and provenance; and new opportunities, for example the success of recommender systems.

Formal challenges: As with the discussion of social network analysis, while the tools for much of this are already formal, it is less clear how, or whether it is valuable, for these to directly connect to user interface models, or whether supporting big data analysis is 'just' another application area. However, there is certainly great opportunity for big data to be used as part of the formal development process; for example, trace data can be mined to propose common interaction patterns, can be used as part of validation or to drive simulations. Also big data about 
applications domains could be used as part of knowledge rich methods (see below).

Autonomy and complexity - Large volumes of data have led to a greater focus on complex algorithms to deal with that data including various forms of machine learning, not least 'deep learning' which has recently been used to master Go (Silver et al., 2016). Many problems, that used to be thought as requiring rich symbolic reasoning, such as translation, are now being tackled using shallow but high volume techniques (Halevy et al., 2009). However, these algorithms are often opaque an issue that was highlighted concerning the very earliest machinelearning-based user interfaces, warning of the potential for potentially unethical or even illegal discrimination and bias (Dix, 1992). As the use of these algorithms have become more common, these dangers have become more apparent leading to the General Data Protection Regulation of the Council of the European Union (2016), which mandates that, for certain forms of critical areas, algorithms need to be able to explain their decisions (Goodman and Flaxman, 2016).

The successes of machine learning have also led to general a resurgence of interest in the potential and dangers of intelligent algorithms. Sometimes this intelligence is used to aid user-driven interactions, and sometimes to act autonomously. The latter have sometimes reached the popular press: for example when scientists called for a ban on autonomous battlefield robots (Hawking, 2015) or when a driver was killed in an self-driving Tesla car (Yadron and Tynan, 2016). In the case of Uber, even when there is a driver in the car, the driver's itinerary and fares are driven by computer algorithms, effectively high-level autonomy.

Formal challenges: The knowledge needed for algorithms to be both intelligent and explicable will draw on expertise form or similar to that found in HCI. Indeed, the UK funding body EPSRC (2016) have identified human-like computing as an important research area and deliberately drawing on cognitive science and human factors as well as artificial intelligence researchers. Some of this will be at a different level then the issues usually studied by those looking at formal methods in HCI, effectively providing application semantics for the user interface. However, the shifts of autonomy do need to be taken into account. There has been work on dynamic function allocation in cockpit and control situations (Hildebrandt and Harrison, 2003), and a few chapters (e.g. 7,15) deal either explicitly or implicitly with more autonomous action sometimes simply at the level of opaque internal transitions.

\subsection{Changing design and development}

New ways of creating software and physical artefacts are altering the processes and people involved in user interface design and hence the notations, tools and analysis techniques needed. 
Maker/hacker culture and mass customization - A new digital DIY culture has emerged made possible by accessible electronics such as Arduino and RaspberryPi and the availability of digital fabrication from fully equipped FabLabs to hobbyist-budget MakerBots. At an industrial-scale, high-budget digital fabrication, such as metal printers, means that the complex and costly spare-parts storage and distribution may soon be a thing of the past replaced by just-in-time printing of everything from spare door handles, to gear boxes. Between the two there is the potential for a new niche of the digital artisan 'modding' consumer products, using open-source 3D-print files, or maybe iTunes-style commercial versions. At both industrial and artisan scale there will certainly be greater scope for individual configuration and semi-bespoke design at a level beyond even today's ideas of mass customisation.

However, if everyone can make their own TV remote or washing machine facia panel, how do you ensure safety and usability of the resulting interfaces. Furthermore, if things do go wrong, who gets sued? For non-critical devices, it may be that we see the HCI equivalent of house makeover television programmes and DIY-style how to books aimed at mass-market. However, for both legal and brand protection products may need to limit acceptable adaptations.

Formal challenges: At first it seems that DIY-culture could not feel further from formal methods; but in fact the need for guaranteed properties on highly configurable interfaces is precisely the kind of problem that formal analysis could address. Specification of usability properties goes back to the earliest days of the FoMHCI community (Dix and Runciman, 1985; Thimbleby and M. D. Harrison, 1990; Dix, 1991) and issues of plasticity and model-based design have been studied for many years (e.g. Coutaz, 2010) and are represented in this book (Chap. 18).

The level of configuration is different from those that are typically addressed (e.g. changes in physical form) and the design audience is somewhat different. It is likely that at least two kinds of design-users need to be addressed: those in commercial enterprises or large open-hardware projects determining the properties required and the range of variations that may be possible; and end-users or those near (e.g. the digital artisan) who are performing modifications with some sort of tool support. The level of formal expertise needed even to understand the outputs of current tool and reasoning support is still far too high, but both the domain specific languages in Chapter 16 and the layered approach in Chapter 14 seem like potential approaches.

Agile and test-driven development - Although there are domains where monolithic development processes dominate, agile development methods are common in many areas, especially web-based systems. Rather like maker culture, at first glance the apparent 'try it and see' feel of agile systems seems at odds with formal development, but in fact agile methodologies are highly disciplined typically combining a strong use-case orientation with test-driven development. Further- 
more, for massive scale systems user-interface development is supported by big data, notable A/B testing (Kohavi et al., 2009; Fisher et al., 2012).

Formal challenges: Although these are very different cultures, there are clear areas where formal methods could make a greater input into agile development. First is use cases, which may already use UML or similar formalisms, and could easily be integrated into an appropriate task modelling framework. This could help with a problem of agile development that it is often hard to keep track of the 'big picture' when constantly creating and deploying incremental change. On the testing side, while some test-based development includes user interfaces, for example, by using headless web browser simulations, this is still a problematic area. Early work has shown that formal property specification could have a place to play, especially in looking at properties that span across individual units of delivery (Bowen and Reeves, 2011). Finally, the volume of data available from large-scale traces of user behaviour is perfect input for performance models such as variants of MHP (Card et al, 1980, 1983), as well as other purposes described previously when looking at big data.

To achieve this would not be without theoretical and practical challenges including addressing presenting methods in ways that are accessible to the ordinary developer, and creating specification methods so that can more easily be addressed to facets of an evolving system.

\section{Formalising interaction: what and how}

Having examined the driving forces from changes in HCI, we now turn to formal methods themselves. We look under four headings.

The first two concern the subject of formal methods in HCI, what they model. The first of these looks at the various actors and entities in the milieu of human interaction with computers, which are currently being modelled and which could be. The second is about different levels of abstraction, generalisation and granularity, the kinds of phenomena that we model and reason about.

The third and fourth headings are more about the process and nature of formal modelling. The first of these looks at the development process for interactive systems and asks when in the process our methods are valuable and who in this process is supported. Finally, we look at how our models work, the kinds of reasoning and modelling we are currently using and how this may need to change or be augmented, especially if we seek to support practical development. 


\subsection{What - actors and entities}

The majority of the earliest work on HCI was focused almost entirely on the direct interaction between a single user and desktop computer or other form of computing device (fig. 1). There was always an interest in the varied stakeholders and other context that surrounded such interactions, but most research, and in particular detailed interaction design and engineering, concerned this dyad. Nor surprisingly this has also been an important theme for formal methods in HCI.

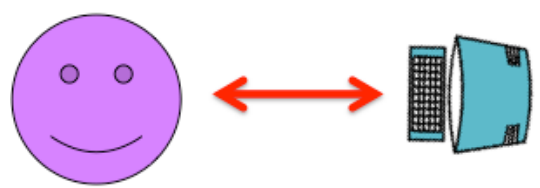

Fig. 1. Human-Computer Interaction: early days - one person, one computer

Of course this dyadic interaction is important, but not the whole story; even today, students need to be constantly reminded to consider the broader context. Figure 2 shows some of the agents and entities in this wider picture. Typically, users do not interact with a single device but several either at different times, or at the same time, for example the mobile phone as 'second screen' while watching television. Users also interact with people and with other aspects of the world: from cars and cats to central heating systems and chemical plants; these interactions may simply set the context for direct computer interaction (e.g. noise as in Chapter 12), but may also in various ways concern the computer, for example finding your way around a city using a smartphone map.

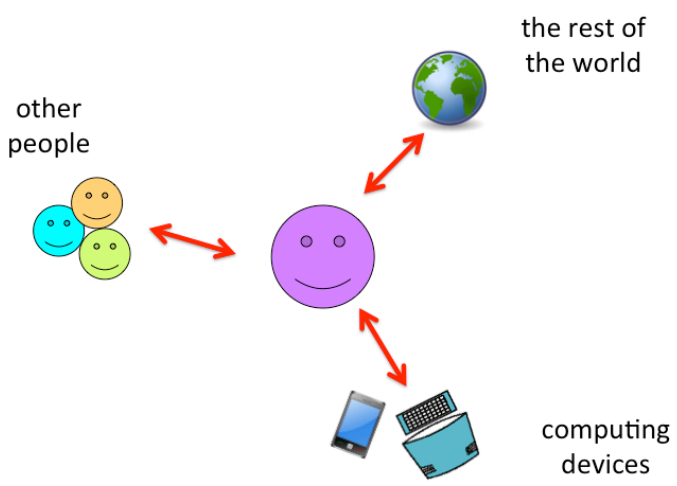

Fig. 2. Human-Computer Interaction: agents and entities 
As well as direct connections, there are indirect interactions: including sensors and actuators on physical objects such as self-driving cars and pervasive computing; computer mediated communication and social networks; virtual elements overlaid on the physical world in augmented reality such as Pokémon Go; computational interfaces embedded into physical appliances such as washing machines; and even computational devices embedded in the user or other people.

Formal challenges: While there is still a strong concentration on direct interactions, the notations and techniques in the chapters in this book do include different elements in this picture. Several include some element of user or task modelling (Chaps 8, 11, 13), building on traditions such as MHP (Card et al, 1980, 1983), ICS (Barnard, 1985) and CCT (Kieras and Polson, 1985), and some include ways to represent multiple actors (Chaps 13,15). As noted under Physicality and embodiment above, there is some work in modelling direct and indirect interactions with the physical environment (chaps 7, 8, 9, 15), but these are still rudimentary.

Even the nature of direct interactions have been changing as noted in discussions of notification based interaction, invisibility and autonomy. There has been a line of work in formal methods dealing with multi-modal interfaces, but now there are fresh challenges, for example dealing with artful interactions such as in crafts or music making and physical movement in the environment.

In general, while formal methods have stepped beyond the single user - single machine dyad, there seems to be substantial room for further work on the richer picture.

\subsection{What - levels of abstraction}

Computer scientists, and especially formalist, love abstractions, and the formalisms in this book and in the community at large vary over a number of dimensions of abstraction.

Granularity - We can view user interaction at a very high/course level in terms of task analysis or workflows; the basic unit at this level might be quite substantial, such as interacting with an app (Chapter 19). We can also look at a very low/fine level of basic motor actions and physical interactions, such as Fitts' Law or physiograms as in Chapter 9. Between these there are many intermediate levels, including the architectural levels in the ARCH/Slinky model. The majority of formal modelling work seems to live in this area where the basic unit of interaction is an abstract button press, or similar action (e.g. Chap. 5).

Formal challenges: This suggests two opportunities for future work: first to expand the focus of methods to encompass more of the higher and lower levels; and second to fit different level of models together to enable reasoning across these. This may not be trivial as interactions regarded as atomic at one level have structure at lower levels. 
Continuity and time - In some ways the finest level of interaction involves continuous action in continuous time. Despite early work on status-event analysis (Dix, 1991, 1991b; Dix and Abowd, 1996) and the TACIT European project (TACIT, 1998; Faconti and Massink, 2002), this is still an understudied area.

Formal challenges: Again there are complex formal issues, but in the broader formal methods community hybrid systems have been studied for many years, so there is clear opportunity to improve coverage. However, as with granularity, the ability to link targeted modelling at different levels seems crucial.

Level of generality - In the earliest strands of formal methods in HCI there was work on notations and methods for specifying specific systems, for example Reisner's (1981) use of BNF and GOMS (Card et al, 1980, 1983); but also work on very abstract models looking at generic properties of all systems, for example the PIE model (Dix and Runciman, 1985) and work on properties of undo (Dix, 1991; Mancini, 1997). In this book, the majority of work is towards the specification of specific systems, but there is also see some work that bridges the extremes of generality including DSL in Chapter 16 and the high level properties in Chapter 14, which both offer ways to create specifications or properties that apply to a subclass of systems. While several chapters use variations of generic systems properties such as predictability of actions or visibility of results, there seems to be little current work directed at this generic level.

Formal challenges: The lack of work at a generic level may be because there are very limited things one can say at this level of generality and the early work saturated the area. However, the work in this volume that operates generically over a sub-class of systems suggests a potential path that allows formalists to work alongside domain experts to create intermediate notations, formal properties, and tools that can then be used by other designers.

Syntax vs semantics - Some philosophers, such as Searle (1997), would argue that by definition anything done in a computer is merely syntax, symbol juggling, and never true semantics as meaning cannot be reduced to rules. However, it is clear that some aspects of computation are more 'semantic' than others. Formal methods often talks about the syntax-semantics distinction, but it is noteworthy that both the Seeheim model (Pfaff and Hagen, 1985) and ARCH/Slinky model (UIMS, 1992; Gram and Cockton, 1996) stop at adaptors/wrappers for application semantics. As noted under granularity, the majority of formal work is focused on the dialogue level, and hence largely about the syntax of interaction, the order of actions and observations, but not their 'meaning'. There are exceptions to this. At a generic level early analysis of undo included use of category theory to model the meaning of undo parameterised over the state semantics of arbitrary systems. At a more specific level task analysis, cognitive models and domain modelling, capture elements of the social, individual and application meaning. The use of ontologies 
and OWL in Chapter 12 is particularly interesting as these are languages of 'semantics'.

Formal challenges: It is possible that rich models of semantics of systems and the environment could become intractable; indeed model checking of user interface specifications already requires some form of abstract interpretation of value domains and faces challenges of combinatorial explosion. However, if formal methods in HCI is to reason about the entire human-computer work system, then there needs to be models of each of the elements, so that the physical properties of the world, the human cognition and action, application 'semantics' and dialogue syntax can be verified to all work together to achieve specified goals. Of course complete models of all of these would be intractable; the greatest challenge will be in determining appropriate levels of detail and abstraction to enable useful results.

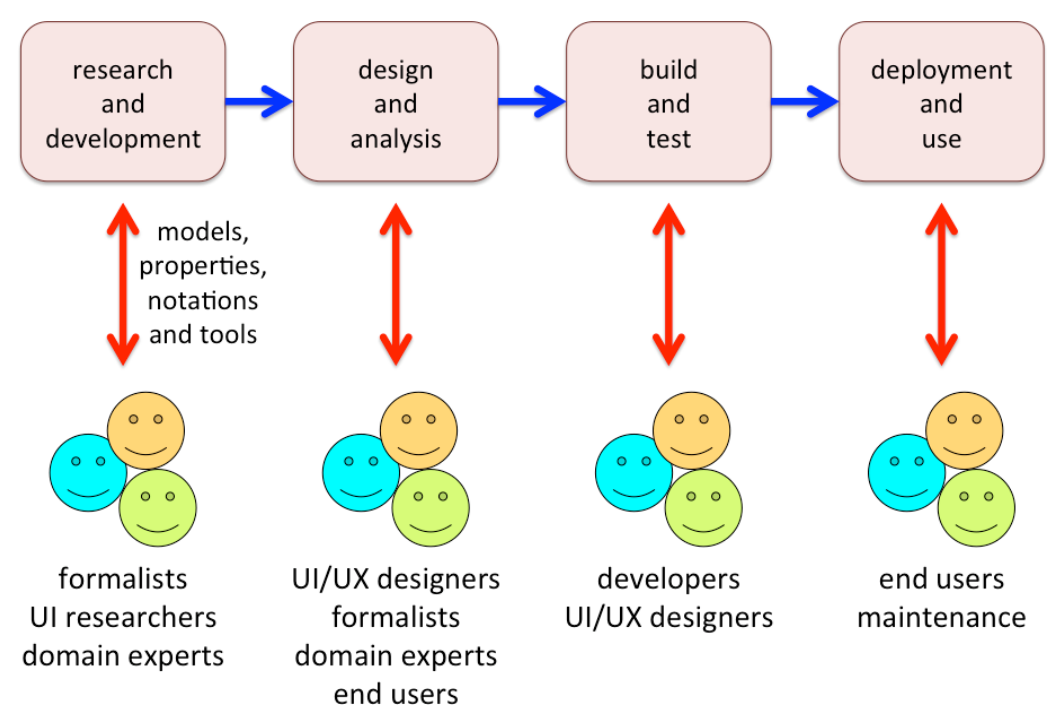

Fig. 3. Design process: processes and people

\subsection{Who and When (and why?)}

Just as we looked at the actors and flows involved in interaction itself, we can consider the actors, activities and products involved in the design process for interactive systems (see Fig. 3). As noted previously, moves towards agile development methods, mean theses stages are likely to be highly iterative including deployment itself, where some web-based systems may have many hundreds of releases per week. 
Very early, indeed before the start of the design process proper, are the formal experts involved in the formulation of notations, properties and tool creation. As noted, this may also include high-level domain experts helping to create more domain specific variants.

During the early stages of the design process for a specific system there are many actors including interaction designers, product designers, user experience specialists; engineers, customers, management, domain experts, ... and hopefully some users! In theory, appropriate notations and tools should help to clarify the design and communicate between stakeholders. The formal outcomes of this stage are detailed specifications, which potentially may be checked using model checkers or other tools.

As the design is turned into code, the formal specification may be translated into executable code; embedded into in-code verification; or used to create test suites.

Finally during use, executable specifications may actually be running in the code, or the user may use manuals that have been in part created, or verified using formal methods (as in Chapter 11). Traces of use may be collected and used as input for analysis tools for future system. During this some systems may be open to end-user modifications or appropriation.

Formal challenges: Although there is potential for formal methods to be useful at many stages in this process, in practice there is little real use beyond limited research case studies and some safety critical systems. Indeed a 2009 ACM Computer Surveys study of industrial use of formal methods all of the systems surveyed had a critical element and only $10 \%$ involved human-computer interaction (Woodcock et al, 2009). The two main barriers are the expertise needed to understand the methods and the time costs of the detailed specification analysis needed even when one has the requisite expertise. Safety critical systems are one of the few areas where the benefits can justify the extreme costs. This leads to three clear challenges: reducing formal expertise, reducing effort, and increasing benefit. These are not new, twenty years ago Clarke and Wing(1996) concluded:

"Success in formal specification can be attributed to notations that are accessible to system designers and to new methodologies for applying these notations effectively" (Clarke and Wing, 1996)

The focus on tool support in several chapters is encouraging as is the use of domain specific properties and languages (Chapters 14,16) as this can help to make the methods more accessible to non-formalists (reducing formal expertise). Ensuring any such tools are usable is also critical, as Chapter 17 emphasises (reducing effort). Indeed it is interesting to see work where the design process is the subject of formal or structured analysis or modelling, including chapter 17's use of Norman's model of action to analyse toolset usability and Bowen and Dittmar's (2016) semi-formal framework for design spaces.

Using toolsets also means that there is greater opportunity to take the same designer input (in the form of task descriptions, interface specifications, etc.) and use it for different purposes, this increasing the benefit. As several chapters were us- 
ing ontologies, RDF and OWL, this suggests the potential for offering some form of expert-system driven guidance (reducing formal expertise), perhaps the outcomes of formal analysis could be used to drive more knowledge-rich explanation systems.

As Chapter 6 points out, designers already create many artefacts such as sketches and low-fidelity prototypes; these could be used more effectively in formal methods in the way Denim (Lin et al, 2000) and subsequent systems did for executable prototypes, that is using computational effort to understand the designer's language. These all become even more important when we consider end-user development, although the use of graph transformations for reconfigurable UIs (Chap. 10) may offer one approach to this.

\subsection{How}

The above take us back to the kinds of formalisms we apply and the ways these might already be changing, or perhaps should change in the future based on some of the challenges we have seen.

Types of reasoning - Many of the chapters in this book use notations and methods that are similar in kind to those found in earlier collections (Thimbleby and Harrison, 1990; Palanque and Paterno, 1997), albeit used in different ways. There is substantial use of textual notations based on sets, functions, predicates and logics; and also more graphical notations including variants of Petri Nets, State Charts and hierarchies for task analysis. A major difference from the early years is that many are subject to some form of automated checking or analysis as well as analysis by hand. Another new development is the use of OWL and similar notations and tools. Unfortunately, as already noted there is still very little use of the mathematics of continuous values or time.

Knowledge rich reasoning -The use of OWL suggests the potential for more knowledge rich reasoning. Traditional mathematics tends to be based on relatively few rules with relatively complex reasoning, in contrast to expert systems in AI with large rule sets (or knowledge bases) and relatively shallow reasoning. However, user interface specification and indeed formal specification in general, tends to already have relatively large specification with relatively simple (possibly automated) analysis. The whole of number theory can be (largely) built from nine Peano axioms, plus a little set theory, even 'toy' interface specifications have more! Furthermore, big data has proved 'unreasonably' effective in using statistical and shallow machine learning techniques to address problems, such as natural language processing, that had formerly been seen as requiring symbolic artificial intelligence (Halevy, 2009). This suggest the potential for using techniques that bring together large data volume knowledge with specifications to address issues 
such as the inclusion of semantics as well as syntax, and the generation of automated design guidance.

Flexible levels of detail - We have seen how different formal notations and techniques operate at different levels of granularity, from workflows on apps to human-motor system analysis. In professional use different levels will be appropriate for different aspects of a system; indeed amongst the conclusions of an analysis of an early successful formal user interface specification case study (Dix, 2002) was the need to be useful (address a real problem) and appropriate (no more detailed than needed), both of which may vary depending on which aspect of the system is under consideration.

Imagine a simple map-based system, that includes buttons to select options, such as satellite imagery vs. schematic, but then uses mouse movement and scroll wheel to drag and zoom the map, when the map is clicked a third-party gazetteer application opens in a pop-up showing additional information about the location. Unless the options buttons are particularly unusual, it will be appropriate to deal with them using a dialogue-level notation such as labelled state transitions (Chaps 11, 15) or ICO (Chap. 17; Palanque, 1992). At this level of specification the map interactions would be abstracted, maybe to separate zoom and scroll functions, or maybe even to a single 'select location'. Similarly, as the gazetteer is pre-existing, we might abstract it to a single "view gazetteer" operation and not attempt to model its interface even at the level of buttons and dialogue. However, we also may want to use a more analysis of the map interactions themselves, perhaps making use of multi-scale Fitts' Law (Guiard et al, 2001).

There are two separate challenges here. First is dealing with systems at multiple levels of detail. This is already studied, for example, Chapter 6 on combining models and Chapters 17 and 18, which have toolsets including notations at different levels; however, this may be more challenging when the notations used involve different paradigms (see below). The second, and perhaps more complex, is when different facets of the system are analysed at different levels so that the specification at certain levels of detail is not 'complete'.

Multiple notations - Many of the chapters in this book use multiple notations. Sometimes this is to deal with different levels as describe above and sometimes because different aspects of the system use different notations, for example user and system models (Chaps 8,11) or physical and digital models (Chap. 9). Even when considering the same aspect at the same level of detail different notations are required for different analysis techniques or tools, a translation process which is usually automated; for example in Chapter 5 a user interface model is translated into a form of Petri Net for execution and in Chapter 16 domain specific languages are translated into a form of UML and into linear temporal logic.

If the underlying paradigms are very close, then this may be relatively unproblematic; however, typically languages have different strengths and information is lost in transforming from one model to another, for example, a single entities in 
one might correspond to several entities in another, or have no correspondence at all. If one notation is semantically richer than the other, then it may be possible to reason across the two by translation, but even then it can be problematic translating and outputs of analysis back. A trivial example of this was the early days of Java Server Pages (JSP) before the development of 'Source Map' files (Oracle. 2003), compiler messages referred to lines in often unintelligible generated Java code. Verifying the connection between notations also requires some sort of explicit or, more often, implicit shared semantics.

Generic descriptions and standards - Whether we are dealing with multiple notations within a single toolset or project, or trying to share artefacts (e.g. UI specifications, traces of user behaviour) between projects, there seems to be a need for different methods, notations and tools to be able to talk to one another. In this book, the nuclear power station case study is specified in some detail in Chapter 4 , but how do we know that the formulation of this in other chapters refers to the 'same' thing? In general, as a community do we need some form of standardised means to share?

At a concrete level, this could be ways to specify elements of interaction such as layouts, behaviours or states, so that it is possible to use these the validate whether two specifications really are talking about the 'same' system. Given the differences between notations and models, the likelihood is that this may need to be partial, perhaps instance based: this particular state has this particular visual appearance and after a specific action modifies to a specified new state. Alternatively it may be possible to have a number of agreed ways of sharing more complex behaviours but limited to particular styles of specification.

At a more abstract level, we could seek semantic models that are not necessarily useful for actual specification (e.g. too verbose or complex), but can be used as a basis for giving semantics for other notations, rather like denotantional semantics does for programing languages (Stoy, 1977). If two notations are given semantics in such a shared semantic model, then it would become possible to assert reliably whether specification in one is equivalent to one in another, or whether a translation algorithm between the two notations is valid.

One suggestion (Dix, 2002) has been that traces could act as a form of universal semantics, both at a concrete level and as a semantic model, as is used by process algebras. The advantage of this is that while different notations vary in how they abstract and parameterise behaviour, the actual realised behaviours are observable and shared. Traces are not without issues, notably continuous vs. discrete time, and even discrete time at different granularities. A similar argument could be made for visual appearance at least for standard control panels with buttons, dropdowns etc. Richer system or interface behaviour are more difficult, although a number of notations are enhanced versions of simpler ones such as labelled transition systems, or Petri Nets; even if it is not possible to have a single interchange model, it maybe possible to have a small selection. 


\section{Summary}

Tables 1 and 2 summarise the principle topics and challenges that have emerged in this chapter; they are quite diverse and offer many opportunities for fruitful future research and real societal and economic impact. From these lists we can bring out a few broader issues.

We need to extend the kinds of issues we approach including users of different abilities; varying devices and infrastructure; physical and semantic interface issues; and privacy. This may include ways to at least partially connect with hard to formalize areas including cognition, emotion and human values.

We need to be able to deal with systems where the computational part is more intelligent, autonomous ad proactive; this includes issues such as notification based systems, internet of things, and robotics.

Some of these new or understudied uses may offer 'easy wins' for FoMHCI, for example, in dealing with the complexities of IoT interactions that are hard for human assessment; or applications to agile methodologies.

We need a range of different levels and kinds of model and the ability to connect these: this includes links between exiting modeling approaches in FoMHCI, and other approaches such as formal argumentation or large knowledge bases.

Applications in safety critical domains are likely to remain a core area of study for FoMHCI as these justify societally and economically the costs of extensive analysis. However, there are opportunities for FoMHCI to become more mainstream both by tackling the 'easy win' areas and seeking ways for formal analysis to become more cost effective and more accessible to domain experts, developers and end users. 
Table 1. HCI trends: summary and formal challenges

\begin{tabular}{|c|c|}
\hline trend & formal challenge \\
\hline \multicolumn{2}{|l|}{ Changing User Interaction } \\
\hline Choice and ubiquity & Importance of diversity (below) \\
\hline Diverse people & $\begin{array}{l}\text { Model-based interfaces to take into account varying abili- } \\
\text { ties }\end{array}$ \\
\hline Diverse devices and contexts & Plastic interfaces beyond screen-size \\
\hline Physicality and embodiment & Modelling beyond syntax layer \\
\hline Really invisible & Radically new models of interaction \\
\hline Experience and values & Linking argumentation to formal modelling \\
\hline Social and personal use & Modelling multiple actors, privacy and provenance \\
\hline Notification-based interaction & $\begin{array}{l}\text { Modelling when uses not in control - matching pace and } \\
\text { timing of notifications to user tasks }\end{array}$ \\
\hline Basic HCI & Better tool support, especially for non-experts \\
\hline \multicolumn{2}{|l|}{ Changing Technology } \\
\hline Vast assemblies & $\begin{array}{l}\text { Application workflows and configuration; } \\
\text { feature interactions }\end{array}$ \\
\hline Big Data & $\begin{array}{l}\text { Use in formal development, e.g. mining trace data; } \\
\text { knowledge rich methods (below) }\end{array}$ \\
\hline Autonomy and complexity & $\begin{array}{l}\text { Human-like computing; dynamic function allocation; } \\
\text { autonomous action beyond internal transitions }\end{array}$ \\
\hline \multicolumn{2}{|l|}{$\begin{array}{l}\text { Changing design and develop- } \\
\text { ment }\end{array}$} \\
\hline $\begin{array}{l}\text { Maker/hacker culture and mass } \\
\text { customization }\end{array}$ & $\begin{array}{l}\text { Guaranteed properties for configurable systems; } \\
\text { tools for near end-users }\end{array}$ \\
\hline Agile and test-driven development & Formalising use cases; generating tests; trace data \\
\hline
\end{tabular}


Table 2. Formalising interaction: summary and formal challenges

\begin{tabular}{|c|c|}
\hline topic & formal challenge/issues \\
\hline \multicolumn{2}{|l|}{ What - Actors, Entities } \\
\hline Actors and entities & $\begin{array}{l}\text { Broading scope of FoMHCI ; modelling users and tasks; } \\
\text { physical aspects; artful interactions }\end{array}$ \\
\hline \multicolumn{2}{|l|}{ What - levels of abstraction } \\
\hline Granularity & $\begin{array}{l}\text { Modelling beyond syntax layer; connecting models at dif- } \\
\text { ferent levels of abstraction }\end{array}$ \\
\hline Continuity and time & Moving beyond discrete event dialogue; hybrid systems \\
\hline Level of generality & Domain-specific generic models \\
\hline Syntax vs semantics & Modelling domain semantics \\
\hline \multicolumn{2}{|l|}{ Who and When (and why?) } \\
\hline Reducing formal expertise & e.g. domain specific notations, expert-system guidance \\
\hline Reducing effort & e.g. toolset development \\
\hline Increasing benefit & e.g. using formal models for multiple purposes \\
\hline \multicolumn{2}{|l|}{ How } \\
\hline Types of reasoning & $\begin{array}{l}\text { Broad range of notations used; increasing usie of automat- } \\
\text { ic analysis; limited knowledge-rich methods; continuity } \\
\text { and time still poor }\end{array}$ \\
\hline Knowledge rich reasoning & $\begin{array}{l}\text { Linking formal specifications and large knowledge-bases; } \\
\text { application semantics; automated design advice }\end{array}$ \\
\hline Flexible levels of detail & $\begin{array}{l}\text { Dealing with multiple levels of detail; working with in- } \\
\text { complete specifications }\end{array}$ \\
\hline Multiple notations & $\begin{array}{l}\text { Translating between notations; verifying connections } \\
\text { (shared semantics) }\end{array}$ \\
\hline Generic descriptions and standards & $\begin{array}{l}\text { Interchange models for specifications, physical layout, } \\
\text { case studies, etc.; shared semantics (e.g. traces) }\end{array}$ \\
\hline
\end{tabular}

\section{References}

Adamczyk P, Bailey B (2004) If not now, when?: the effects of interruption at different moments within task execution. In Proceedings of the SIGCHI Conference on Human Factors in Computing Systems (CHI '04). ACM, New York, NY, USA, 271-278. doi: $10.1145 / 985692.985727$

Bailey N, Konstan J (2006) On the need for attention-aware systems: Measuring effects of interruption on task performance, error rate, and affective state, Computers in Human Behavior, 22(4):685-708, doi: 10.1016/j.chb.2005.12.009 
Barnard P (1985) Interacting cognitive subsystems: a psycholinguistic approach to shortterm memory. In A. Ellis, editor, Progress in the Psychology of Language, volume 2, chapter 6. Lawrence Erlbaum Associates, Hove

Bowen J. Reeves S (2011) UI-driven test-first development of interactive systems. In Proceedings of the 3rd ACM SIGCHI symposium on Engineering interactive computing systems (EICS '11). ACM, New York, NY, USA, 165-174. doi: $10.1145 / 1996461.1996515$

Bowen J, Dittmar A (2016) A semi-formal framework for describing interaction design spaces. In Proceedings of the 8th ACM SIGCHI Symposium on Engineering Interactive Computing Systems (EICS '16). ACM, New York, NY, USA, 229-238. DOI: $10.1145 / 2933242.2933247$

Bruegger P (2011). uMove: A wholistic framework to design and implement ubiquitous computing systems supporting user's activity and situation. PhD Thesis, University of Fribourg, Switzerland. http://doc.rero.ch/record/24442

Card S, Moran T, Newell A (1980) The keystroke-level model for user performance with interactive systems. Communications of the ACM, 23:396-410

Card S, Moran T, Newell A (1983) The Psychology of Human Computer Interaction. Lawrence Erlbaum Associates, Hillsdale, New Jersey

Carter T, Seah S, Long B. Drinkwater B, Subramanian S (2013) UltraHaptics: multi-point mid-air haptic feedback for touch surfaces. In Proceedings of the 26th annual ACM symposium on User interface software and technology (UIST '13). ACM, New York, NY, USA, 505-514. doi: 10.1145/2501988.2502018.

Citizens Advice Bureau (2013) 22\% don't have basic banking services needed to deal with Universal Credit. Retrieved 29/01/2014, from http://www.citizensadvice.org.uk/index/pressoffice/press_index/press_office20131105.h tm

Clarke E, Wing J (1996) Formal methods: state of the art and future directions. ACM Comput. Surv. 28, 4 (December 1996), 626-643. doi: 10.1145/242223.242257

Cockton G (2004) Value-centred HCI. In Proceedings of the third Nordic conference on Human-computer interaction (NordiCHI '04). ACM, New York, NY, USA, 149-160. doi: $10.1145 / 1028014.1028038$

Council of the European Union (2016). Position of the council on general data protection regulation. 8 April 2016. http://www.europarl.europa.eu/sed/doc/news/ document/CONS_CONS(2016)05418(REV1)_EN.docx.

Coutaz, J (2010) User Interface Plasticity: Model Driven Engineering to the Limit! In Engineering Interactive Computing Systems (EICS 2010), ACM. p 1-8.

Czerwinski M, Horvitz E, Wilhite S (2004) A diary study of task switching and interruptions. In Proceedings of the SIGCHI Conference on Human Factors in Computing Systems (CHI '04). ACM, New York, NY, USA, 175-182. doi: 10.1145/985692.985715. 
Dewsbury G, Ballard D (2014) DTA: The Dependability Telecare Assessment tool - The Person-Centred Telecare Assessment. Bourne, UK: gdewsbury. http://www.gdewsbury.com/dta/

Dix A, Runciman C (1985) Abstract models of interactive systems. People and Computers: Designing the Interface, Ed. P. J. \&. S. Cook. Cambridge University Press. p 13-22. http://alandix.com/academic/papers/hci88/

Dix A (1990) Information processing, context and privacy. Human-Computer Interaction INTERACT'90, Ed. D. G. D. Diaper G. Cockton \& B. Shakel. North-Holland. p 15-20.

Dix A (1991) Formal methods for interactive systems. London: Academic Press. http://www.hiraeth.com/books/formal/

Dix A (1991b) Status and events: static and dynamic properties of interactive systems. Proceedings of the Eurographics Seminar: Formal Methods in Computer Graphics, Ed. D. A. Duce. Marina di Carrara, Italy, http://www.comp.lancs.ac.uk/computing/users/dixa/papers/euro91/euro91.html

Dix A (1992) Human issues in the use of pattern recognition techniques. In Neural Networks and Pattern Recognition in Human Computer Interaction Eds. R. Beale and J. Finlay. Ellis Horwood. p http://alandix.com/academic/papers/neuro92/neuro92.html

Dix A (1992b) Pace and interaction. Proceedings of HCI'92: People and Computers VII, Eds. A. Monk, D. Diaper and M. Harrison. Cambridge University Press. 193-207. http://alandix.com/academic/papers/pace/

Dix A, Abowd G (1996) Modelling status and event behaviour of interactive systems. Software Engineering Journal, 11(6):334-346. http://alandix.com/academic/papers/euro91/

Dix A (2002) Towards a Ubiquitous Semantics of Interaction: phenomenology, scenarios and traces. Interactive Systems. Design, Specification, and Verification 9th International Workshop, DSV-IS 2002. P. Forbrig, Q. Limbourg, B. Urban, J. Vanderdonckt (eds). Rostock, Germany, June 2002. Springer, LNCS 2545, p 238-252. http://alandix.com/academic/papers/dsvis2002/

Dix A (2002) Formal Methods in HCI: a Success Story - why it works and how to reproduce it. Lancaster University, UK. http://alandix.com/academic/papers/formal-2002/

Dix A, Ramduny-Ellis D, Wilkinson J (2004) Trigger Analysis - understanding broken tasks. Chapter 19 in The Handbook of Task Analysis for Human-Computer Interaction. D. Diaper \& N. Stanton (eds.). Lawrence Erlbaum Associates, 2004. p 381-400 http://www.hcibook.com/alan/papers/triggers2002

Dix A, Sheridan J, Reeves S, Benford S, O'Malley C (2005) Formalising Performative Interaction. Proceedings of DSVIS'2005. (Newcastle, UK, 13-15 July 2005). Springer, LNCS 3941, p 15-25. http://www.hcibook.com/alan/papers/DSVIS2005---performance/ 
Dix A, Leavesley J (2015) Learning Analytics for the Academic: An Action Perspective. In Journal of Universal Computer Science (JUCS), 21(1):48-65 http://www.hcibook.com/alan/papers/JUCS-action-analytics-2015/

Dix A (2016) Human computer interaction, foundations and new paradigms, Journal of Visual Languages \& Computing, in press. doi: 10.1016/j.jvlc.2016.04.001

Duncan D, Hoekstra A, Wilcox B (2012) Digital Devices, Distraction, and Student Performance: Does In-Class Cell Phone Use Reduce Learning? Astronomy Education Review 11(1), doi: 10.3847/AER2012011

Ellis C (1994) Goal Based Workflow Systems. International Journal of Collaborative Computing, 1(1):61-86

EPSRC (2016) Human-Like Computing Report of a Workshop held on 17 \& 18 February 2016, Bristol, UK. https://www.epsrc.ac.uk/newsevents/pubs/humanlikecomputing/

Eslambolchilar P (2006) Making sense of interaction using a model-based approach. PhD thesis, Hamilton Institute, National University of Ireland, NUIM, Ireland

Faconti G, Massink M (2002) A reference framework for continuous interaction, International Journal Universal Access in the Information Society, 1(4):237-251. Springer Verlag

Fatah gen Schieck A, Kostakos V, Penn A, O'Neill E, Kindberg T, Stanton Fraser D, Jones $\mathrm{T}$ (2006) Design tools for pervasive computing in urban environments. In: van Leeuwen, JPT and P, HJ, (eds.) Innovations in design and decision support systems in architecture and urban planning. (pp. 467-486). Springer: Dordrecht, Netherlands.

Fisher D, DeLine R, Czerwinski M, Drucker S (2012) Interactions with big data analytics. interactions 19(3):50-59. doi:10.1145/2168931.2168943

Goodman B, Flaxman S (2016) EU regulations on algorithmic decision-making and a "right to explanation". Presented at 2016 ICML Workshop on Human Interpretability in Machine Learning (WHI 2016), New York, NY. http://arxiv.org/abs/1606.08813v1

Gram C, Cockton G (eds) (1996) Design principles for interactive software. London: Chapman \& Hall

Guiard Y, Bourgeois F, Mottet D, Beaudouin-Lafon M (2001) Beyond the 10-bit Barrier: Fitts' Law in Multi-Scale Electronic Worlds. People and Computers XV-Interaction without Frontiers: Joint Proceedings of HCI 2001 and IHM 2001, Springer, London. p 573-587. doi: 10.1007/978-1-4471-0353-0_36

Halevy A, Norvig P, Pereira F (2009) The Unreasonable Effectiveness of Data. IEEE Intelligent Systems, 24(2):8-12, doi: 10.1109/MIS.2009.36

Harper R, Rodden T, Rogers Y, Sellen A (eds) (2008) Being Human: Human-Computer Interaction in the year 2020. Microsoft Research. http://research.microsoft.com/enus/um/cambridge/projects/hci2020/ 
Hawking S, Musk E, Wozniak S et al (2015) Autonomous Weapons: an Open Letter from AI \& Robotics Researchers. Future of Life Institute. http://futureoflife.org/AI/open_letter_autonomous_weapons

Henrich J, Heine S, Norenzayan A (2010) The weirdest people in the world? Behavioral and Brain Sciences, 33(2-3):61-83. doi: 10.1017/S0140525X0999152X

Hildebrandt M, Harrison M (2003) Putting time (back) into Dynamic Function Allocation. In Proceedings of the 47th Annual Meeting of the Human Factors and Ergonomics Society

Ishii H (2003) Tangible bits: designing the seamless interface between people, bits, and atoms. In Proceedings of the 8th international conference on Intelligent user interfaces (IUI '03). ACM, New York, NY, USA, 3-3. doi: 10.1145/604045.604048

Hong J, Landay J (2004) An architecture for privacy-sensitive ubiquitous computing. In Proceedings of the 2nd international conference on Mobile systems, applications, and services (MobiSys '04). ACM, New York, NY, USA, p 177-189. doi: $10.1145 / 990064.990087$

Keim D, Kohlhammer J, Ellis G, Mansmann F (2010) Mastering the information agesolving problems with visual analytics. Eurographics Association, Goslar, Germany. http://www.vismaster.eu/wp-content/uploads/2010/11/VisMaster-book-lowres.pdf

Kieras D, Polson P (1985) An approach to the formal analysis of user complexity. International Journal of Man-Machine Studies, 22:365-94

Kohavi R, Longbotham R, Sommerfield D, Henne R (2009) Controlled experiments on the web: survey and practical guide. Data Min. Knowl. Discov. 18(1):140-181. doi:10.1007/s10618-008-0114-1

Langheinrich M (2002) A Privacy Awareness System for Ubiquitous Computing Environments. In Proceedings of the 4th international conference on Ubiquitous Computing (UbiComp '02), Gaetano Borriello and Lars Erik Holmquist (Eds.). Springer-Verlag, London, UK, UK, p 237-245.

Lin J, Newman M, Hong J, Landay J (2000) DENIM: finding a tighter fit between tools and practice for Web site design. In Proceedings of the SIGCHI conference on Human Factors in Computing Systems (CHI '00). ACM, New York, USA, p 510-517. doi: $10.1145 / 332040.332486$

Mancini R (1997) Modelling Interactive Computing by Exploiting the Undo. Dottorato di Ricerca in Informatica, IX-97-5, Università degli Studi di Roma "La Sapienza". http://www.hcibook.net/people/Roberta/

McCoy B (2016) Digital Distractions in the Classroom Phase II: Student Classroom Use of Digital Devices for Non-Class Related Purposes. Journal of Media Education 7(1):5-32. http://en.calameo.com/read/00009178915b8f5b352ba

Meixner G, Paternó F, Vanderdonckt J (2011) Past, Present, and Future of Model-Based User Interface Development. i-com, 10(3):2-11 
Norman D (2005) Emotional Design. Basic Books

Oracle (2003) JSR-045: Debugging Support for Other Languages. JSRs: Java Specification Requests. https://jcp.org/en/jsr/detail?id=45

Palanque P (1992) Modélisation par Objets Coopératifs Interactifs d'interfaces hommemachine dirigées par l'utilisateur. $\mathrm{PhD}$ : Toulouse I

Palanque P, Paterno F (eds) (1997) Formal Methods in Human-Computer Interaction. London, Springer-Verlag

Pallotta V, Bruegger P, Hirsbrunner B (2008) Kinetic User Interfaces: Physical Embodied Interaction with Mobile Ubiquitous Computing Systems. In S. Kouadri-Mostefaoui, M. Maamar, P. Giaglis (eds.) Advances in Ubiquitous Computing: Future Paradigms and Directions, IGI Global Publishing, p 201-228. ISBN: 978-1-599-04840-6

Pfaff G, Hagen P (eds) (1985) Seeheim Workshop on User Interface Management Systems, Springer- Verlag, Berlin

Reisner P (1981) Formal Grammar and Human Factors Design of an Interactive Graphics System. IEEE Trans. Softw. Eng. 7(2):229-240. doi: 10.1109/TSE.1981.234520

Searle J (1997) The Mystery of Consciousness. Granta Books, London.

Shackel B (1959) Ergonomics for a computer, Design, 120:36-39. (available at http://www.idemployee.id.tue.nl/g.w.m.rauterberg/presentations/shackel-1959.PDF)

Sherman J (2013) Half of benefit claimants lack skill to complete online forms. The Times. London: Nov. 6 2013. http://www.thetimes.co.uk/tto/news/uk/article3914355.ece

Silver D et al (2016) Mastering the game of Go with deep neural networks and tree search. Nature. 529:484-489. Xdoi: 10.1038/nature16961

Stoy J (1977) Denotational Semantics: The Scott-Strachey Approach to Programming Language Semantics. MIT Press, Cambridge, Massachusetts

Sufrin B (1982) Formal specification of a display-oriented text editor, Science of Computer Programming, 1:157-202, North Holland Publishing Co.

TACIT (1998-2002) Theory and Applications of Continuous Interaction Techniques. EU

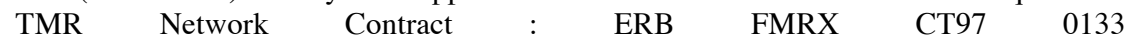
http://www.webalice.it/giorgio.faconti/TACIT/TACITweb/doclist.html

Thimbleby H, Harrison M (eds) (1990) Formal Methods in Human-Computer Interaction. Cambridge University Press

Thimbleby H (2007) Using the Fitts law with state transition systems to find optimal task timings. In: Proc. of Second Intnl. Workshop on Formal Methods for Interactive Systems, FMIS2007 
http://www.dcs.qmul.ac.uk/research/imc/hum/fmis2007/preproceedings/FMIS2007prepr oceedings.pdf

Thomas J, Cook K (2005) Illuminating the Path: Research and Development Agenda for Visual Analytics. IEEE Press

Turchi T, Malizia A (2016) A Human-Centred Tangible approach to learning Computational Thinking. EAI Endorsed Transactions on Ambient Systems 16(9):e6. doi: 10.4108/eai.23-8-2016.151641

UIMS (1992) A Metamodel for the Runtime Architecture of An Interactive System. The UIMS Developers Workshop, SIGCHI Bulletin, 24(1):32-37, ACM, January, 1992. doi: $10.1145 / 142394.142401$

Weiser M (1991) The computer of the 21st century. Scientific American, 265(3):66-75, September 1991

Whittington P, Dogan H (2016) A SmartDisability Framework: enhancing user interaction. Proceedings of the 2016 British HCI Conference

Woodcock J, Larsen P, Bicarregui J, Fitzgerald J (2009) Formal methods: Practice and experience. ACM Comput. Surv. 41(4):19 (October 2009), doi: 10.1145/1592434.1592436

Wurdel M (2011) An Integrated Formal Task Specification Method for Smart Environments. University of Rostock. isbn: 978-3-8325-2948-2

Yadron D, Tynan D (2016) Tesla driver dies in first fatal crash while using autopilot mode. The Guardian, Friday 1 July 2016 